\title{
Optimal dispatch of active and reactive power using cuckoo search method
}

\author{
G. R. Goyal ${ }^{1}$, H. D. Mehta ${ }^{2}$ \\ P.G. Student, Electrical, L. D. College of Engineering, Ahmedabad, India ${ }^{1}$ \\ Associate Professor, Electrical, L. D. College of Engineering, Ahmedabad, India ${ }^{2}$
}

\begin{abstract}
This paper deals with application of evolutionary algorithm (EA) to solve optimal power flow problem in an efficient manner. In this paper a new approach using cuckoo search (CS) method is proposed for solving OPF problem by optimal setting of control variables. Cuckoo search method is a bio-inspired algorithm based on brooding behaviour of cuckoo birds. This algorithm can search for a global solution using multiple paths. Different objective functions as fuel cost minimization and power loss minimization has been considered for optimal active \& reactive power dispatch respectively. The proposed method is implemented and evaluated on the IEEE 30-bus system. The simulation results of the proposed approach are compared to others those reported in the literature. The results demonstrate the potential of the proposed approach and show its effectiveness and robustness to solve the OPF problem.
\end{abstract}

Keywords: Optimal power dispatch, Cuckoo search method, evolutionary algorithm.

\section{INTRODUCTION}

In present scenario power engineers are facing a main problem of handling the available power in an effective manner to cope-up with the speedy growing demand of consumers. In power system operation and planning, OPF is a best option to handle such problems. Optimal active and reactive power flow is concerned with the minimization of power losses, economic generation and minimization of other environmental harmful effects. Here in OPF solutions, objective is achieved through optimal adjustment of system control variables while satisfying the system constraints at same time. Transformer-tap setting, real power generated and generator bus voltages are the control variables. As equality and inequality constraints power flow equation and limits of control variables are considered.

Optimal power problem was started by Carpentier in 1960s. Today computer based techniques are used for the optimization but behind the computer there is always an algorithm that works. Various algorithms are available in mathematics to find the optimum solution of any non linear function. These are classified as conventional and evolutionary. Some of conventional methods like Gradient Method [1], Interior Point Method [2], linear programming [3] has been proposed for the reactive power optimization successfully, but some difficulties are associated with these methods $[3,10]$. OPF problem is not a linear one and it is non- differential too [15]. So the problem with conventional methods while solving the OPF problem is the solution may got trapped by local optimum value [9]. Other than this, problem with conventional methods is if the state variables are defined in very short range than solution becomes infeasible.

To overcome these problems Evolutionary Algorithms are introduced as, Stochastic Algorithms e.g. GA, PSO, ACO and SA etc. These all are nature inspired algorithms. These further classified as heuristic and meta-heuristic algorithms. Alan Turing was the first who used heuristic algorithms in 1948. After that, 1960s and 1970s were the two decades of development of Evolutionary Algorithms. Today these are very popular to solve the real-world optimization problems. These algorithms are equally useful in Optimal Power flow problems [3-5]. Genetic Algorithm (GA) [3,4], Particle swarm optimization (PSO) [6,13], Differential Evolution (DE) [9,10] have been proposed for reactive power optimization, voltage control and minimization of cost of active and reactive power both as an objective function.

In recent a new evolutionary algorithm cuckoo search (CS) has been introduced. This algorithm is inspired by brooding behaviour of cuckoos and based on levy flights. It can take care of optimality on rough and discontinuous surface. It can handle integer and discrete optimization problem. It is easy to implement because of less number of control variables $[14,20]$.

In $[15,16]$, the CS algorithm is used as an optimization tool for minimizing cost of power generation while maintaining the dependent variables.

In this paper, a novel CS-based approach is proposed to solve the problem of optimal dispatch of active and reactive power. The problem is formulated as a nonlinear function with equality and inequality constraints. In this study, different objectives are considered such as minimizing the power losses and cost of power generation. The proposed approach has been examined and tested on the standard IEEE 30-bus test system. The potential and effectiveness of the proposed approach are demonstrated. Additionally, the results are compared with other methods those reported in the literature.

\section{PROBLEM FORMULATION}

The objective of optimal power flow is to identify the control variables which minimize the objective function. This is formulated mathematically as follows:

\subsection{Problem objectives}

In this paper following objective are considered: 


\subsubsection{Minimization of system power losses}

Here minimization of system real power losses, Ploss (MW) is considered as one of the objective function. Reactive power control variables are adjusted at their optimal settings which can minimize the system real power losses. The power loss function is as follows [9]:

$$
f=\text { Ploss }=\sum_{k=1}^{N L} g_{k}\left[\left|V_{i}\right|^{2}+\left|V_{j}\right|^{2}-2\left|V_{i} \| V_{j}\right| \cos \left(\delta_{i}-\delta_{j}\right)\right]----(1)
$$

It is the loss formula at kth line connected between ith and jth bus. Here, NL is the number of transmission lines; gk is the conductance of the $\mathrm{kth}$ line; $\mathrm{Vi}$ and $\mathrm{Vj}$ are the voltage magnitude at bus end $\mathrm{i}$ and $\mathrm{j}$ of the line, respectively, and $\delta \mathrm{i}$ and $\delta \mathrm{j}$ are the voltage phase angles at the end buses $\mathrm{i}$ and $\mathrm{j}$.

\subsubsection{Minimization of fuel cost with real power output}

The fuel cost of each fossil fuel fired generator can be expressed as a single quadratic function but the cost function has discontinuities corresponding to change of fuels [5]. Therefore, it is more appropriate to represent the cost function with piecewise quadratic functions. The total fuel cost in terms of real power output can be expressed as [13]:

$$
\begin{aligned}
& f(P g)=\sum_{i=1}^{N G} C_{i} \\
& f(P g)=\sum_{i=1}^{N G} a_{i} P g_{i}^{2}+b_{i} P g_{i}+c_{i} \$ / h---(
\end{aligned}
$$

\subsection{System Constraints}

In this paper following equality and inequality constraints are considered:

\subsubsection{Equality Constraints}

$$
\sum_{i=1}^{N G} P g_{i}-\sum_{i=1}^{N B} P d_{i}-\text { Ploss }=0---(3)
$$

$P g-P d-V i \sum_{j=1}^{N B} V j[G i j \cos (\delta i-\delta j)+B i j \sin (\delta i-\delta j)]-(4)$

Above equations are the power balance equation and power flow equation. Where $\mathrm{i}=1$, . .,NB; $\mathrm{NB}$ is the number of buses, $\mathrm{Pg}$ is the active power generated, $\mathrm{Pd}$ is the active power load, $\mathrm{Gij}$ and $\mathrm{Bij}$ are the transfer conductance and susceptance between bus $\mathrm{i}$ and bus $\mathrm{j}$, respectively.

\subsubsection{Inequality Constraints}

1. Generator Constraints: Generator voltages are varied in between their upper and lower bounds:

$$
V g_{i}^{\min } \leq V g_{i} \leq V g_{i}^{\max }--(5)(i=1,2 \ldots N G)
$$

2. Transformer Constraints: transformer tap-setting are bounds as follows:

$T_{i}^{\min } \leq T_{i} \leq T_{i}^{\max }--(6)(i=1,2 \ldots . N T)$
3. Generator Constraints: generator power output is varied in its upper and lower range:

$P g_{i}^{\min } \leq P g_{i} \leq P g_{i}^{\max }--(7)(i=1,2 \ldots N G)$

4. Security Constraints: these includes the constraints of voltage level at load buses as follows:

$V_{l i}^{\min } \leq V_{l i} \leq V_{l i}^{\max }--(8)(i=1,2, . . N L)$

\section{CUCKOO SEARCH METHOD}

\subsection{Overview}

Cuckoo search method is one of the nature inspired metaheuristic algorithms. It was developed in 2009 by Xin- She Yang and Suesh Deb. Cuckoo search method is based on brood parasitism of cuckoo birds. These birds have a different kind of brooding behaviour. It lays its eggs in communal nests. There are basically three types of brooding parasitism: First is Intra-specific brooding second is cooperative brooding and third is nest takeover. It depends because if any host bird discovers the align egg it may get rid of these align eggs or simply abandon its nest and build a new nest. Based on the breeding behaviour of cuckoos an algorithm works in order to find most suitable host nest.

So this algorithm is designed for maximization of problem but it can be used for minimization equally. Here, fitness function is the objective function which is to be optimized and quality of solution is directly proportional to the value of objective function or fitness function.

\subsection{Levy Flights}

Levy flight is named for French mathematician Paul Levy; it shows the typical characteristics of flight behaviour of birds. Birds search for food in a random manner. Their search path is made up of step-by-step random walks or flights. Levy flights are random walks where the step size/ length are distributed according to the heavy tailed distribution. If walks are defined in a space of dimension greater than one, steps will be taken in random directions. It is based on the current location and transition probability to the next location.

$$
\alpha=0.01 * \mathrm{~s}(\mathrm{X}-\mathrm{Gbest})
$$

\subsection{Mantegna Algorithm}

Mantegna algorithm developed by R. Mantegna, is used to produce random numbers as per symmetric Levy distribution, symmetric means that steps can be positive or negative. In the algorithm while generating random numbers uniform distribution is taken into account with zero means and standard variance is calculated by following formula:

$$
\sigma_{u}=\left\{\frac{\Gamma(1+\beta) \sin (\pi \beta / 2)}{\Gamma[(1+\beta) / 2] \beta 2^{(\beta-1) / 2}}\right\}^{1 / \beta}---(10)
$$

\section{$3.4 \quad$ Process of Cuckoo Search}

For implementation of Cuckoo algorithm we assume that there are certain numbers of cuckoos. It is assumed that each cuckoo lays single egg at one time in randomly 
chosen nest. It is also assumed that there is fixed number of nests available as host. During the survival competition some of the eggs may get destroyed. The survived cuckoos immigrate to a new \& better environment and start reproduction and laying new eggs. The ultimate aim of this algorithm is to use the new and better solutions to replace the not-so-good solutions present in the nests.

On behalf of the Cuckoo algorithm's theory we have the following idealized rules:

1. The number of host nests and Cuckoos remains fixed.

2. Each Cuckoo lays single egg at a time and dumps it in a randomly chosen nest, one egg in one nest.

3. The best nests with high quality eggs will be carried over to the next generation.

4. The probability of egg laid by cuckoo is discovered by host nest i.e. abundance probability, is $\mathrm{Pa}$ in the range of $[0,1]$.

As the all evolutionary algorithms, Cuckoo algorithm starts with some initial population. Their efforts to survive constitute the basis of cuckoo search algorithm.

Levy flight provides a random walk whose step size is decided by Levy Distribution, it have infinite variance with infinite means.

$\operatorname{Levy}(\lambda)=\mathrm{t}-\lambda$------- (11) $\quad 1<\lambda \leq 3$

It can be implemented in Cuckoo search as if algorithm generates new solutions $\mathrm{Xt}+1$ from the present set of solutions $\mathrm{Xt}$, for any given problem using following equation

$\mathrm{Xt}+1=\mathrm{Xt}+\alpha \oplus_{\text {Levy }}(\lambda)$---- (12) $\quad$ where, $\alpha>0$

The step size $\alpha$ is adjustable as per requirement of problem. These steps essentially form a random walk process. In the above equation some new solutions will be generated by Levy walk around the present best solution Gbest.

\subsection{Flow chart}

A step by step process of algorithm for cuckoo search method is given in fig.- 3

\section{SIMULATION STUDY \& RESULTS}

\subsection{IEEE- 30 bus system}

The proposed approach is implemented and tested on a test bed IEEE- 30 bus 6-gen system, which is shown in the figure- 4 and the system data is considered [9]. This system is consists of 48 branches, 6 generators-buses and 22 load-buses. Here, Bus-1 is considered as slack bus. PVbuses: BUS 2,5,8,11,13 and others are PQ-buses.

\subsection{Optimal solution obtained by CS}

To demonstrate the effectiveness of the above method in OPF problem two cases with different objectives are considered and implemented on IEEE- 30 bus, results are as follows

Case-1 Minimization of active power losses

Case-2 Minimization of fuel cost

\subsubsection{Case-1 (Minimization of active power loss)}

In the first case, the proposed algorithm is run with objective of minimization of active power losses. As mentioned above, the generator bus voltage and tap setting are varied in their range (voltage in 0.95-1.05 and tap setting in 0.90-1.1. The convergence characteristic of algorithm is shown in fig.-1. The algorithm reaches a minimum loss of $7.52 \mathrm{MW}$. The optimal setting of control variables is given in table- 1 .

\begin{tabular}{|c|c|c|c|}
\hline $\begin{array}{c}\text { S. } \\
\text { No. }\end{array}$ & Parameter & $\begin{array}{c}\text { Initial } \\
\text { value }\end{array}$ & Optimal value \\
\hline 1 & V1 & 1.06 & 1.02545 \\
\hline 2 & V2 & 1.04 & 0.98319 \\
\hline 3 & V5 & 1.01 & 0.96042 \\
\hline 4 & V8 & 1.01 & 1.04504 \\
\hline 5 & V11 & 1.05 & 0.95080 \\
\hline 6 & V13 & 1.05 & 0.98743 \\
\hline 7 & T11 & 1.078 & 0.96128 \\
\hline 8 & T12 & 1.069 & 1.06129 \\
\hline 9 & T15 & 1.032 & 0.97297 \\
\hline 10 & T36 & 1.068 & 1.00345 \\
\hline 11 & Loss (MW) & $\mathbf{1 0 . 3 8}$ & $\mathbf{7 . 5 2}$ \\
\hline
\end{tabular}

Table-1 Optimal setting of control variable for case-1

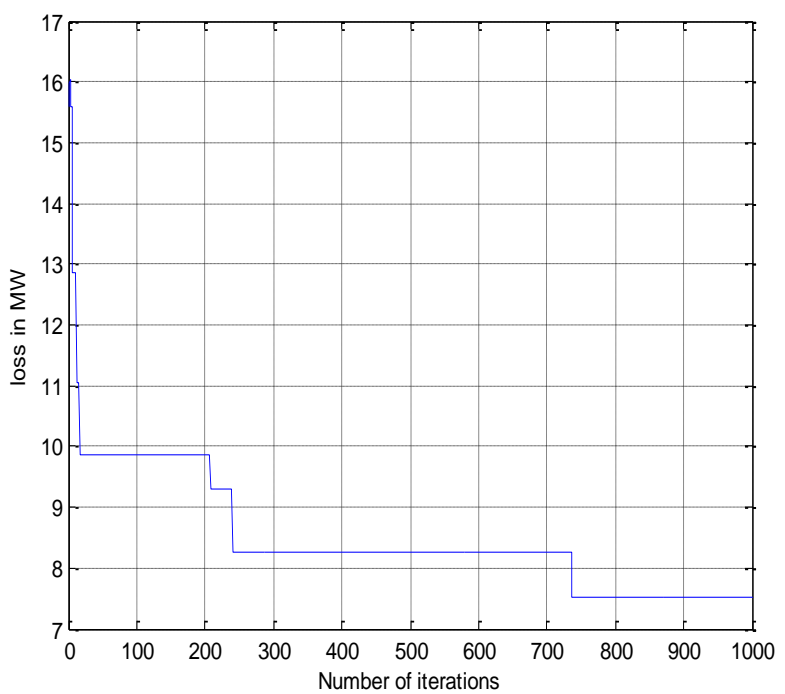

Fig. -1 Graph for fitness Vs No. of iterations for case-1

\section{Case-2 (Minimization of fuel cost)}

In the second case, the proposed algorithm is run with minimization of fuel cost of active power generation as the objective function. As mentioned above, the real power outputs of the generators are varied in their range. The convergence characteristic of algorithm is shown in fig.-2. The algorithm reaches a minimum cost of $831.85 \$ / \mathrm{h}$. The optimal setting of control variables is given in table- 2 . 


\begin{tabular}{|c|c|c|c|}
\hline $\begin{array}{c}\text { S. } \\
\text { No }\end{array}$ & Parameter & $\begin{array}{c}\text { Initial } \\
\text { value }\end{array}$ & $\begin{array}{c}\text { Optimal } \\
\text { value }\end{array}$ \\
\hline 1 & $\operatorname{Pg} 1$ & 104.62 & 151.72 \\
\hline 2 & $\operatorname{Pg} 2$ & 80.00 & 45.12 \\
\hline 3 & $\operatorname{Pg} 5$ & 50.00 & 33.76 \\
\hline 4 & $\operatorname{Pg} 8$ & 20.00 & 25.35 \\
\hline 5 & $\operatorname{Pg} 11$ & 20.00 & 24.06 \\
\hline 6 & $\operatorname{Pg} 13$ & 20.00 & 14.62 \\
\hline 7 & $\begin{array}{c}\text { Total Cost of gen. } \\
(\$ / h)\end{array}$ & $\mathbf{9 1 6 . 2 9}$ & $\mathbf{8 3 1 . 8 5}$ \\
\hline
\end{tabular}

Table-2 Optimal setting of control variable for case-2

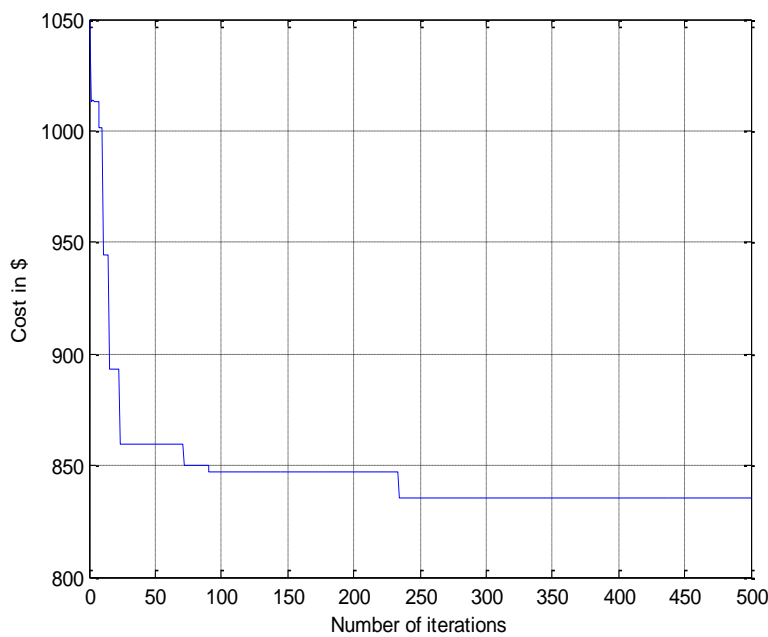

Fig.- 2 Graph fitness Vs No. of iterations for case-2

The results of cuckoo search method are compared with other evolutionary methods with same objectives as per literature.

\begin{tabular}{|c|l|c|}
\hline $\begin{array}{c}\text { S. } \\
\text { No. }\end{array}$ & \multicolumn{1}{|c|}{ Method } & $\begin{array}{c}\text { \% Minimization } \\
\text { of power loss }\end{array}$ \\
\hline 1 & Gradient Method [1] & 9.4 \\
\hline 2 & Interior Point Method [2] & 6.0 \\
\hline 3 & Genetic Algorithm [4] & 16.23 \\
\hline 4 & Differential Evolution [9] & 22.03 \\
\hline 5 & Proposed Method & 27.6 \\
\hline
\end{tabular}

Table-3 Comparison of different methods for case-1

\begin{tabular}{|c|l|c|}
\hline $\begin{array}{c}\text { S. } \\
\text { No. }\end{array}$ & \multicolumn{1}{|c|}{ Method } & $\begin{array}{c}\text { \% Minimization } \\
\text { of fuel cost }\end{array}$ \\
\hline 1 & Gradient Method [1] & 3.54 \\
\hline 2 & Particle Swarm [12] & 3.12 \\
\hline 3 & Genetic Algorithm [5] & 7.25 \\
\hline 4 & Proposed Method & 9.21 \\
\hline
\end{tabular}

Table-4 Comparison of different methods for case-2

\section{CONCLUSION}

In this paper, Cuckoo search method (CS) has been developed, implemented on MATLAB-2013 and successfully applied to solve optimal active and reactive power dispatch problem. The OPF problem has been formulated as a constrained optimization problem where two separate objective functions have been considered to minimize power losses and cost of power generation. The proposed approach have been tested and examined on the standard IEEE 30-bus test system. The above approach resulted with minimization of fuel cost \& power losses by $9.21 \%$ and $27.6 \%$ respectively in comparison of power flow solutions. The simulation results demonstrate the effectiveness and robustness of the proposed algorithm to solve OPF problem.

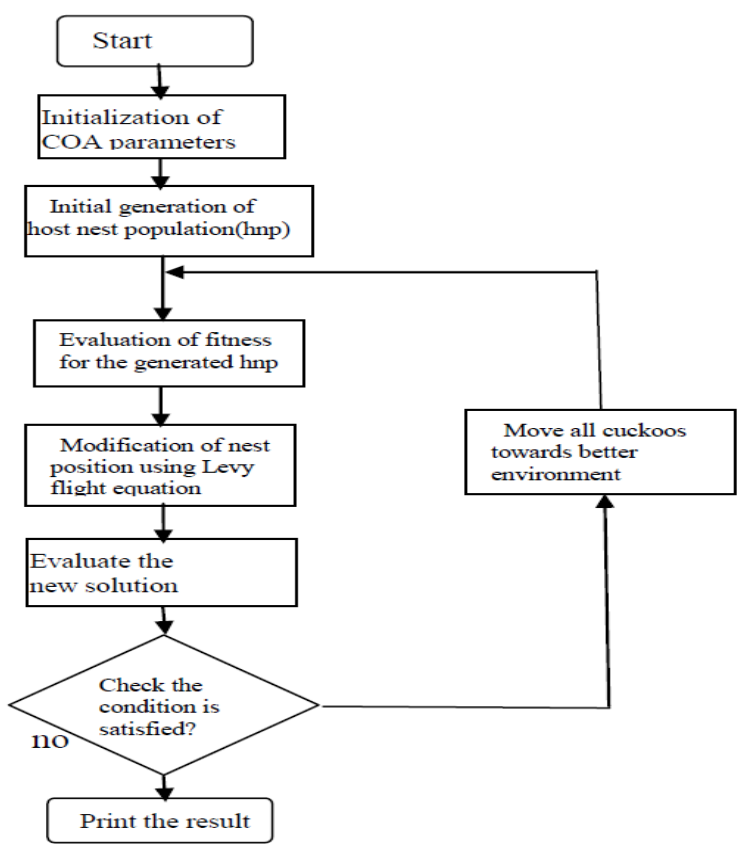

Fig.-3 Flow Chart for Cuckoo search Algorithm

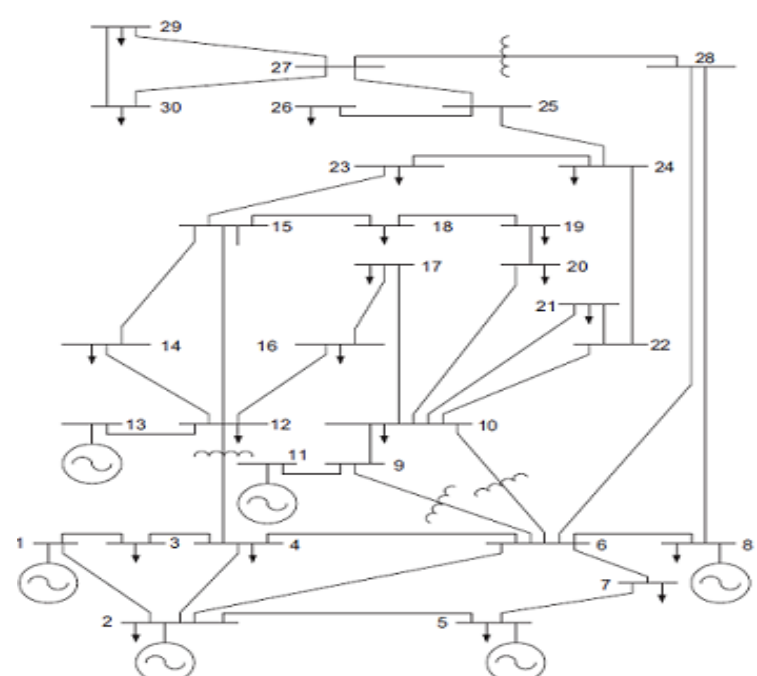

Fig. -4 Single line diagram for IEEE- 30 bus system

\section{REFERENCES}

[1] K.Y. Lee, Y.M. Park and J.L. Ortiz, "A united approach to real and reactive power dispatch", IEEE Transaction on Power Apparatus and systems, Vol. PAS-104, No.5, 1991.

[2] Sergio Granville, "Optimal Reactive Power Dispatch through Interior Point Method", IEEE Transaction on Power Systems, vol. 5, No. 1, February 1985. 
[3] Kenji Iba, "Reactive Power Optimization by Genetic Algorithm", IEEE Transactions on Power Systems, Vol. 9, No, 2, May 1994.

[4] Q H Wu and J T Ma, "Power system optimal reactive power dispatch using evolutionary programming”, IEEE Transactions on Power System, Vol. 10. No. 3, August 1995.

[5] Kwang Y. Lee and Frank F. Yang, "Optimal Reactive Power Planning Using Evolutionary Algorithms: A Comparative Study for Evolutionary Programming, Evolutionary Strategy, Genetic Algorithm, and Linear Programming", IEEE Transactions on Power Systems, Vol. 13, No. 1, February 1998.

[6] H. Yoshida, Y. Fukuyama, K. Kawata, S. Takayama, Y. Nakanishi, "A particle swarm optimization for reactive power and voltage control considering voltage security assessment", IEEE Trans on Power Systems, Vol. 15, No.4, pp 1232-1239, November 2001

[7] P. Subburaj, N. Sudha, Dr. K. Ramar, Dr. L. Ganesan, "Optimum Reactive Power Dispatch Using Genetic Algorithm”, Academic Open Internet Journal, Volume 21, 2007, ISSN 1311-4360.

[8] S.A. Jumaat, Ismail Musirin, Muhammad Murtadha Othman, and Hazlie Mokhlis, "PSO Based Technique for Loss Minimization Considering Voltage Profile and Cost Function", International Power Engineering and Optimization Conference, Malaysia: 6-7 June 2011, 978-1-4577-0354-6.

[9] A.A. Abou El Ela, M.A. Abido, S.R. Spea, "Differential evolution algorithm for optimal reactive power dispatch", ELSEVIERElectric Power Systems Research 81 (2011) 458-464.

[10] Bhushan Wane, "Optimization of reactive power for line loss reduction and voltage profile improvement using Differential Evolution Algorithm”, IJERSTE, ISSN: 2319-7463 Vol. 2 Issue 12, December-2013, and PP: (29-34).

[11] S. Sakthivel, A. Subramanian, S. Gajendran, P.V. Selvan, "Reactive Power Reserve Management by Using Improved Particle Swarm Optimization Algorithm", IJCER, Volume, 03, Issue 4, April 2013.

[12] Mahalakshmi G. and Bhavani M, "Power System Reactive Power Optimization Using DPSO, IEEE International Conference on Innovations in Engineering and Technology, Volume 3, Special Issue 3, March 2014.

[13] D. P. Kothari, Y.S. Brar, H.P. Singh, "Combined active and reactive power dispatch Using particle swarm optimization", Proceedings of ISITE, 2014 (295-304).

[14] Yang X.S. and Deb S, "Engineering Optimisation by Cuckoo Search", IJRMNO, Vol. 1, No. 4, 330-343 (2010).

[15] A.Hima Bindu, Dr. M. Damodar Reddy, "Economic Load Dispatch Using Cuckoo Search Algorithm”, IJERA, Vol. 3, Issue 4, Jul-Aug 2013, pp. 498-502

[16] Adriane B.S.Serapião, "Cuckoo Search for Solving Economic Dispatch Load Problem", Scientific Research, Intelligent Control and Automation, 2013, 4, 385-390

[17] Pinar civicioglu and Erkan Besdok, "A conceptual comparison of the Cuckoo-search, particle swarm optimization, differential evolution and artificial bee colony algorithms", Artif Intell Rev DOI 10.1007/s10462-011-9276-0, 06 July-2011.

[18] M.RamaMohana Rao, A.V.Naresh Babu, "Optimal power flow using Cuckoo optimization algorithm”, International Journal of Advanced Research in Electrical, Electronics and Instrumentation Engineering, Vol.2, Issue 9, September 2013, ISSN (Online): 2278 - 8875

[19] Adil Hashmi, Divya Gupta, Nishant Goel et al, "Comparative Study of Bio-inspired algorithms for Unconstrained Optimization Problems", AEECE2013, ISBN: 978-981-07-6935-2 doi: 10.3850/ 978-981-07-6935-2_28.

[20] Dr. T. Govindaraj and S. Udayakumar, "Optimal Reactive Power Planning and Real Power Loss Minimization Using Cuckoo Search Algorithm", International journal of innovative research in electrical, electronics, instrumentation and control engineering Vol. 2 , issue 2, February 2014, ISSN (Print) $2321-5526$ 